\title{
Extended Acceleration in Slot Gaps and Pulsar High-Energy Emission
}

\author{
Alex G. Muslimov ${ }^{1} \&$ Alice K. Harding ${ }^{2}$
}

\begin{abstract}
We revise the physics of primary electron acceleration in the "slot gap" (SG) above the pulsar polar caps (PCs), a regime originally proposed by Arons and Scharlemann (1979) in their electrodynamic model of pulsar PCs. We employ the standard definition of the $\mathrm{SG}$ as a pair-free space between the last open field lines and the boundary of the pair plasma column which is expected to develop above the bulk of the PC. The rationale for our revision is that the proper treatment of primary acceleration within the pulsar SGs should take into account the effect of the narrow geometry of the gap on the electrodynamics within the gap and also to include the effect of inertial frame dragging on the particle acceleration. We show that the accelerating electric field within the gap, being significantly boosted by the effect of frame dragging, becomes reduced because of the gap geometry by a factor proportional to the square of the SG width. The combination of the effects of frame dragging and geometrical screening in the gap region naturally gives rise to a regime of extended acceleration, that is not limited to "favorably curved" field lines as in earlier models, and the possibility of multiple-pair production by curvature photons at very high altitudes, up to several stellar radii. We present our estimates of the characteristic SG thickness across the PC, energetics of primaries accelerated within the gap, high-energy bolometric luminosities emitted from the high altitudes in the gaps, and maximum heating luminosities produced by positrons returning from the elevated pair fronts. The estimated theoretical high-energy luminosities are in good agreement with the corresponding empirical relationships for $\gamma$-ray pulsars. We illustrate the results of our modeling of the pair cascades and $\gamma$-ray emission from the high altitudes in the SG for the Crab pulsar. The combination of the frame-dragging field and high-altitude $\mathrm{SG}$ emission enables both acceleration at the smaller inclination angles and a larger emission beam, both necessary to produce widely-spaced double-peaked profiles.
\end{abstract}

Subject headings: pulsars: general - radiation mechanisms: nonthermal - stars: neutron - $\gamma$-rays: stars

\footnotetext{
${ }^{1}$ ManTech International Corporation, Lexington Park, MD 20653

${ }^{2}$ Laboratory of High Energy Astrophysics, NASA/Goddard Space Flight Center, Greenbelt, MD 20771
} 


\section{INTRODUCTION}

There has been a steadily growing number of rotation-powered pulsars with emission detected at high energies, following the success of the Compton Gamma-Ray Observatory (Thompson 2001) and detections by ROSAT, ASCA, RXTE and most recently by Chandra and XMM (Becker \& Aschenbach 2002). With the number of pulsars having detected emission above $1 \mathrm{keV}$ now approaching several dozen, high-energy radiation seems to be a common feature. Although the theory of acceleration and high-energy emission in pulsars has been studied for over twenty-five years, the origin of the pulsed non-thermal emission is a question that remains unsettled. The observations to date have not been able to clearly distinguish between an emission site at the magnetic poles (Daugherty \& Harding 1996, hereafter DH96) and emission from the outer magnetospheric gaps (Cheng, Ho \& Ruderman 1986, Hirotani \& Shibata 2001). Complicating the problem is the fact that both models have some unresolved difficulties. In the case of polar cap (PC) models, while the energetics and pair-cascade spectrum have had success in reproducing the observations, the predicted beam size of radiation emitted near the neutron star (NS) surface is too small to produce the wide pulse profiles that are observed. However, Arons (1983, hereafter A83) first noted the possibility of a high-altitude acceleration region or "slot gap" (SG) near the PC rim, based on the finding of Arons \& Scharlemann (1979, hereafter AS79) that the pair formation front $(\mathrm{PFF})$, above which the accelerating field is screened, occurs at increasingly higher altitude as the magnetic colatitude approaches the last open field line where the electric field vanishes. The SG is a characteristic feature that is unavoidable in any PC space-charge limited flow model. In the last few years, a self-consistent model of PC acceleration has been developed, which includes effects of general relativity (Harding \& Muslimov 1998, hereafter HM98) and screening by pairs (Harding \& Muslimov 2001, hereafter HM01; Harding \& Muslimov 2002, hereafter HM02). HM98 concentrated on acceleration in the interior regions of the PC, but their calculation confirmed that a $\mathrm{SG}$ also forms near the $\mathrm{PC}$ rim in the presence of inertial frame dragging. Interestingly, since the magnitude, and longitudinal and radial dependences of the accelerating electric field used in HM98 are different from those in the model of AS79, in the calculation of HM98 the PFF is curving up more abruptly to form the inner boundary of a much narrower SG.

The present paper is motivated by the fact that, despite the attractive possibility of primary acceleration within the $S G$ region of a pulsar inner magnetosphere (AS79), the electrodynamics and energetics of the $\mathrm{SG}$ acceleration remain to a large extent unexplored beyond the original model of AS79. What we know about SG acceleration is mostly based on a classical model of AS79 and further developments by A83 and Arons (1996, hereafter A96), and it has never been investigated in any other electrodynamic model thus far. However, we believe that within the general-relativistic model of space-charge limited flow from the pulsar PC (Muslimov \& Tsygan, 1992, hereafter MT92) the acceleration properties within the SG will significantly differ from those implied by the SG originally proposed by AS79. The SG model of AS79 and A83 produced effective acceleration only for large inclination angles and only on "favorably curved" field lines, i.e. those field lines curving toward the rotation axis. The frame-dragging acceleration dominates 
at most pulsar periods at small inclination angles, and operates over the entire PC (i.e. it is not restricted to favorably curved field lines). Besides this simple update of the underlying electrodynamic model, we incorporate an additional effect of screening of the electric field within the PC slot, a narrow space between the last open field lines and lines confining the pair plasma column generated at relatively low altitudes above the PC surface. This enhanced screening is caused by taking into account a second conducting boundary formed by the pair plasma column very near the last open field line. The $E_{\|}$within the $\mathrm{SG}$ is greatly reduced by the proximity of two conducting boundaries and is well-known from the electrodynamics in narrow tubes and cones with conducting surfaces (Landau \& Lifshitz 1984). Ironically, this effect of screening by two closely spaced conducting boundaries was overlooked in original calculations of the SG acceleration, in which only the conducting boundary at the last open field line was taken into account. To estimate the field in the SG, A83 simply applied the solution for the accelerating electric field in the absence of the pair plasma, assuming screening only from the last open field line boundary.

The major advantage of acceleration within the SG is the possibility of establishing the curvature radiation (CR) PFF and generation of $\gamma$-ray emission at high altitudes. The need to locate the generation of pair cascades and $\gamma$-ray emission at high altitude above the PC was demonstrated by Daugherty \& Harding (1996, hereafter DH96), and later also by Rudak et al. (2002) who numerically modeled the pair formation, high-energy radiation spectra and light curves at different altitudes in pulsars. They found that the shape of the cascade spectrum and high-energy cutoff, due to magnetic pair creation, for the Vela pulsar best matches the observed spectrum for emission at altitudes around 1-2 stellar radii above the surface. Furthermore, the wide $\gamma$-ray profiles observed can be produced in PC models only if the emission beam opening angle is comparable to the inclination angle. High altitude emission along flaring field lines would enable larger high energy beams. DH96 approached the problem by trying to reproduce the observed spectra and light curves of the Vela pulsar by assuming a simple parameterized model of the accelerating electric field. However, the physical origin of that accelerating field as well as possible regimes of acceleration and energetics for different pulsar spin periods and magnetic fields could not be addressed in that approach. Also the details of such modeling, such as the pair-formation altitudes, hardness of the spectrum, and widths of the high-energy pulses, will also depend on the regime of acceleration of primaries within the SG. In his study of high-energy emission from the SG, A96 came to the conclusion that the SG energetics, as determined by a potential which did not include the frame-dragging effect, is not enough to account for the observed $\gamma$-ray luminosities and spectra of the Crab and Vela pulsars. Furthermore, he investigated only the CR from the primary particles accelerating in the SG which results in a spectrum that does not match the softer observed spectra of $\gamma$-ray pulsars. In this paper, we additionally consider the emission from the high-altitude pair cascades above the PFF that bounds the inner edge of the $\mathrm{SG}$. We derive the general relativistic accelerating electric field in the case of the SG to include the effect of screening caused by the slot geometry, and present our calculations of pair cascades at high altitudes (at a few stellar radii above the surface), $\gamma$ - ray spectra, and $\gamma$ - ray light 
curves. We provide simple expressions for the $\gamma$-ray and heating luminosities, and discuss possible constraints on the Crab pulsar viewing angles and obliquities.

The paper is organized as follows. In $\S 2$ we discuss the electrodynamics within the SG regions of pulsars. We present the electric potential drop in $\S 2.1$, calculate the energetics of primaries in $\S 2.2$, estimate the characteristic gap thickness in $\S 2.3$, and derive $\gamma$-ray luminosities per solid angle emitted by the SGs as well as heating luminosities in $\S 2.4$. In $\S 3$ we discuss the main results of our numerical cascade simulations. Finally, in $\S 4$. we discuss our main results and draw our principal conclusions.

\section{Electrodynamics of Slot Gap}

The SG model for the emission region of a pulsar was originally proposed by AS79, who were the first to observe that in a space-charge limited flow the process of pair formation may significantly vary across the PC. For example, in their electrodynamic model, the pair formation surface tends to curve up and become asymptotically tangent to the field lines near the boundary of the polar tube in its half with the so-called "favorably curved" field lines. This occurs simply because the acceleration of electrons in this region is not sufficient to produce pairs at low altitude, leaving a SG around the column of pair plasma. This model was described in detail by A83, and also overviewed by Arons (1981) and A96. The very concept of SG acceleration, and its potential impact on pair production, radio and high-energy emission from pulsars, was a significant finding. However, the original SG treatment failed to produce a viable model of pulsar high-energy emission. One reason is that the calculation of electron acceleration within the SG was inconsistent with the acceleration and pair formation in rest of polar flux tube region. In this paper we discuss and calculate the effect that we believe makes the regime of SG acceleration very feasible and attractive.

Figure 1 schematically illustrates the geometry of the open field lines at the PC. The extended SG region is squeezed between the pair plasma column and closed magnetospheric region filled with plasma. The ring-like structure on the top indicates the elevated pair formation site.

\subsection{Electric Potential Drop within the Slot Gap}

The SG region is bounded by the last open field lines and the lines with the magnetic colatitude $\left(1-\Delta \xi_{\mathrm{SG}}\right)$ times smaller, where $\Delta \xi_{\mathrm{SG}}$ is the latitudinal gap thickness in units of $\xi$ $\left(\xi=\theta / \theta_{0}\right.$ is dimensionless colatitude of a PC field line, and $\theta_{0} \approx(\Omega R / c f(1))^{1 / 2}$ is the footpoint of the last open field line). Let us introduce the field line separating the $\mathrm{SG}$ into two halves and having the magnetic colatitude $\theta_{0, \mathrm{SG}}=\theta_{0}\left(1-\Delta \xi_{\mathrm{SG}} / 2\right)$, and denote the innermost half-space of a SG as region I and the outermost half-space as region II. Then the magnetic field lines within 
each of these regions can be described by the colatitudes

$$
\begin{aligned}
\theta_{\mathrm{SG}}(\eta)= & \theta_{0, \mathrm{SG}}\left[\eta \frac{f(1)}{f(\eta)}\right]^{1 / 2}\left[1-\frac{1}{2} \Delta \xi_{\mathrm{SG}} \xi_{*}\right]= \\
& \theta_{0}\left[\eta \frac{f(1)}{f(\eta)}\right]^{1 / 2}\left[1-\frac{1}{2} \Delta \xi_{\mathrm{SG}}\left(1+\xi_{*}\right)\right], \quad \xi_{*} \in[1,0] \quad \text { for region I }
\end{aligned}
$$

and

$$
\begin{aligned}
\theta_{\mathrm{SG}}(\eta)= & \theta_{0, \mathrm{SG}}\left[\eta \frac{f(1)}{f(\eta)}\right]^{1 / 2}\left[1+\frac{1}{2} \Delta \xi_{\mathrm{SG}} \xi_{*}\right]= \\
& \theta_{0}\left[\eta \frac{f(1)}{f(\eta)}\right]^{1 / 2}\left[1-\frac{1}{2} \Delta \xi_{\mathrm{SG}}\left(1-\xi_{*}\right)\right], \quad \xi_{*} \in[0,1] \quad \text { for region II, }
\end{aligned}
$$

respectively.

Here $\xi_{*}$ is the dimensionless colatitude of field lines bounded by the SG regions I and II and which is counted from the field line that separates those regions, $\eta=r / R$ is the dimensionless radial coordinate, and $f(\eta)$ is the correction factor for the dipole component of the magnetic field in a Schwarzchild metric (see MT92 for details).

We shall also introduce the SG half-width in the longitudinal direction scaled to the PC surface, $\delta_{\mathrm{SG}}=(1 / 2) r_{\mathrm{pc}} \Delta \xi_{\mathrm{SG}}$, where $r_{\mathrm{pc}}=R \theta_{0}$ is the $\mathrm{PC}$ radius. In a small-angle approximation (which is perfect for the SG case) the Goldreich-Julian (GJ) charge density and the charge density of primary electrons read

$$
\rho_{\mathrm{GJ}}=-\frac{\Omega B_{0}}{2 \pi c \alpha \eta^{3}} \frac{f(\eta)}{f(1)}\left[\left(1-\frac{\kappa}{\eta^{3}}\right) \cos \chi+\frac{3}{2} \theta_{\mathrm{SG}}(\eta) H(\eta) \sin \chi \cos \phi_{\mathrm{pc}}\right]
$$

and

$$
\rho=-\frac{\Omega B_{0}}{2 \pi c \alpha \eta^{3}} \frac{f(\eta)}{f(1)}\left[(1-\kappa) \cos \chi+\frac{3}{2} \theta_{\mathrm{SG}}(1) H(1) \sin \chi \cos \phi_{\mathrm{pc}}\right]
$$

respectively.

Here $\Omega=2 \pi / P, P$ is the pulsar spin period, $B_{0}$ is the surface value of the magnetic field strength at magnetic pole, $\alpha$ is the redshift function, $\kappa=\left(r_{\mathrm{g}} / R\right)\left(I / I_{0}\right) \approx 0.15 I_{45} / R_{6}^{3}$ is the general relativistic parameter entering the frame-dragging effect, $r_{\mathrm{g}}$ is the NS gravitational radius, $I_{45}=I / 10^{45} \mathrm{~g} \cdot \mathrm{cm}^{2}, R_{6}=R / 10^{6} \mathrm{~cm}, I$ is the NS moment of inertia, $I_{0}=M R^{2}, M$ and $R$ are, respectively, NS mass and radius, $H$ is a relativistic correction factor of order one, $\chi$ is the pulsar obliquity, and $\phi_{\mathrm{pc}}$ is the magnetic azimuthal angle (see MT92 for details).

For high enough altitudes within the SG, i.e. for $\eta-1 \gg \delta_{\mathrm{SG}}$, and in a small-angle approximation, the general-relativistic Poisson's equation (see also the derivation of eq. [37] in MT92),

$$
\nabla \cdot\left(\frac{1}{\alpha} \nabla \Phi\right)=-4 \pi\left(\rho-\rho_{\mathrm{GJ}}\right)
$$


translates into

$$
\frac{1}{\alpha \theta \eta^{2}}\left[\frac{\partial}{\partial \theta}\left(\theta \frac{\partial}{\partial \theta}\right)+\frac{1}{\theta} \frac{\partial^{2}}{\partial \phi_{\mathrm{pc}}^{2}}\right] \Phi\left(\eta, \theta, \phi_{\mathrm{pc}}\right)=-2 \frac{1}{\alpha \eta^{3}} \frac{f(\eta)}{f(1)} \Phi_{0}\left(\mathcal{A} \cos \chi+\mathcal{B} \sin \chi \cos \phi_{\mathrm{pc}}\right),
$$

where

$$
\begin{gathered}
\mathcal{A}=\kappa\left(1-1 / \eta^{3}\right) \\
\mathcal{B}=(3 / 2)\left[H(\eta) \theta_{\mathrm{SG}}(\eta)-H(1) \theta_{\mathrm{SG}}(1)\right]
\end{gathered}
$$

By employing expressions (1), (2) and changing variables from $\theta$ to $\xi_{*}$ in eq. (6), and also assuming the approximation $\Delta \xi_{\mathrm{sG}} \ll 1$ we get

$$
\left(\frac{1}{\nu_{\mathrm{SG}}} \frac{\partial^{2}}{\partial \xi_{*}^{2}}+\frac{\partial^{2}}{\partial \phi_{\mathrm{pc}}^{2}}\right) \Phi\left(\eta, \xi_{*}, \phi_{\mathrm{pc}}\right)=-2 \Phi_{0} \theta_{0}^{2}\left(\mathcal{A} \cos \chi+\mathcal{B} \sin \chi \cos \phi_{\mathrm{pc}}\right)
$$

where

$$
\nu_{\mathrm{SG}}=\frac{1}{4} \Delta \xi_{\mathrm{SG}}^{2}=\left(\frac{\delta_{\mathrm{SG}}}{r_{\mathrm{pc}}}\right)^{2}
$$

is the screening factor originating from the gap geometry. Also in eq. (9) $\Phi_{0}=(\Omega R / c) B_{0} R$, which is the characteristic value of the maximum potential drop in a vacuum solution (see Deutsch 1955).

Assuming that the SG surfaces are equipotential $\left(\Phi\left[\xi_{*}=1\right]=0\right)$, the solution of $(9)$ for the electric potential within regions I and II of a SG can be written as

$$
\Phi\left(\eta, \xi_{*}, \phi_{\mathrm{pc}}\right)=\Phi_{0} \theta_{0}^{2}\left\{\nu_{\mathrm{sG}} \mathcal{A}\left(1-\xi_{*}^{2}\right) \cos \chi+2 \mathcal{B}\left[1-\frac{\cosh \left(\sqrt{\nu_{\mathrm{SG}}} \xi_{*}\right)}{\cosh \left(\sqrt{\nu_{\mathrm{SG}}}\right)}\right] \sin \chi \cos \phi_{\mathrm{pc}}\right\} .
$$

Since $\nu_{\mathrm{SG}} \ll 1$, we can write that $2\left[1-\cosh \left(\sqrt{\nu_{\mathrm{SG}}} \xi_{*}\right) / \cosh \left(\sqrt{\nu_{\mathrm{SG}}}\right)\right] \approx \nu_{\mathrm{SG}}\left(1-\xi_{*}^{2}\right)$. Hence, eq. (11), after substitution the explicit expressions for $\mathcal{A}$ and $\mathcal{B}$, translates into

$$
\begin{aligned}
\Phi\left(\eta, \xi_{*}, \phi_{\mathrm{pc}}\right)= & \Phi_{0} \theta_{0}^{2} \nu_{\mathrm{sG}}\left[\kappa\left(1-\frac{1}{\eta^{3}}\right) \cos \chi+\frac{3}{2} \theta_{0, \mathrm{SG}} H(1) \times\right. \\
& \left.\left(\frac{H(\eta)}{H(1)} \sqrt{\eta \frac{f(1)}{f(\eta)}}-1\right) \sin \chi \cos \phi_{\mathrm{pc}}\right]\left(1-\xi_{*}^{2}\right) .
\end{aligned}
$$

Note that the angular size of the SG limits the maximum lengthscale of the angular part of Laplacian and naturally enters the solution of Poisson's equation (see MT92 for details). The factor $\nu_{\mathrm{SG}}$, to be evaluated in Section 2.3 , is proportional to the square of the SG thickness $\Delta \xi_{\mathrm{SG}}$. Thus, the additional screening introduced by the conducting boundary at the PFF results in a reduction of the potential $\Phi(\eta)$ and the associated $E_{\|}$in the SG. It is important that from the electrodynamic point of view, we treat the $\mathrm{SG}$ in exact same way we generally treat the whole region of open field lines of the PC. Thus, the physics of screening effect within the SG is akin to the physics of screening of the vacuum electric field within the open field line region of pulsar by the geometry of the region itself. 


\subsection{Primary Energetics within the SG and Emerging $\gamma$ - Ray Flux}

Let us write the general formula for the luminosity of primaries accelerating in a SG,

$$
L_{\mathrm{prim}}\left(\eta_{\mathrm{PFF}}\right)=\alpha c \int_{S_{\mathrm{SG}}\left(\eta_{\mathrm{PFF}}\right)}|\rho(\eta)| \Phi(\eta) d S(\eta),
$$

where the integration is over the surface cut in a sphere at the radial distance $\eta_{\mathrm{PFF}}$ by the $\mathrm{SG}$ boundaries, and $\eta_{\mathrm{PFF}}$ refers to the elevated PFF within the SG region.

In eq. (13) $d S$ is the element of a spherical surface at radial distance $\eta$. After inserting expressions (4) and (12) into eq. (13), we get

$$
L_{\mathrm{prim}}=f_{\mathrm{SG}} L_{\mathrm{sd}}
$$

where

$$
\begin{aligned}
f_{\mathrm{SG}}= & \nu_{\mathrm{SG}} \Delta \xi_{\mathrm{SG}}\left\{\kappa(1-\kappa)\left(1-\frac{1}{\eta^{3}}\right) \cos ^{2} \chi+\right. \\
& \left.\frac{9}{8} \theta_{0}^{2} H^{2}(1)\left[\frac{H(\eta)}{H(1)} \sqrt{\eta \frac{f(1)}{f(\eta)}}-1\right] \sin ^{2} \chi\right\}_{\left.\right|_{\eta=\eta_{\mathrm{PFF}}},},
\end{aligned}
$$

is the efficiency of converting the spin-down power into the power of primary particles within the $\mathrm{SG}$, and $L_{\mathrm{sd}}=\Omega^{4} B_{0}^{2} R^{6} / 6 c^{3} f^{2}(1)$ is the pulsar spin-down luminosity, where $B_{0} / f(1)$ is the surface value of the magnetic field strength corrected for the "gravitational redshift".

Note that the efficiency $f_{\mathrm{sG}}$ is a function of pulsar parameters $B$ and $P$ (via $B, P$-dependence of $\nu_{\mathrm{SG}}$ and $\Delta \xi_{\mathrm{SG}}$, , so the luminosity of a primary beam scales as $L_{\text {prim }} \propto L_{\mathrm{sd}}^{\alpha}$, where $\alpha=1 / 7$ and $4 / 7$ for $B \lesssim 0.1 B_{\mathrm{cr}}$ and $\gtrsim 0.1 B_{\mathrm{cr}}$, respectively (see $\S 2.4$ for details).

\subsection{Estimate of Slot Gap Longitudinal Thickness}

In this paragraph we estimate the characteristic scale of a SG over the colatitude $\xi$-coordinate. We shall discriminate between two cases where the local magnetic field $B \lesssim 0.1 B_{\text {cr }}$ and $B \gtrsim 0.1 B_{\text {cr }}$, respectively, where $B_{\mathrm{cr}}=4.413 \cdot 10^{13} \mathrm{G}$ is the quantizing value of the magnetic field strength. For $B<0.1 B_{\mathrm{cr}}$, the attenuation length of the CR photons is determined by the condition $\epsilon B^{\prime} \sin \theta_{\mathrm{kB}} \gtrsim 0.2$, whereas for $B>0.1 B_{\mathrm{cr}}$ it is determined by the threshold condition $\epsilon \sin \theta_{\mathrm{kB}}>2$, where $\epsilon$ is the photon energy in units of $m_{\mathrm{e}} c^{2}, B^{\prime}=B / B_{\mathrm{cr}}$, and $\theta_{\mathrm{kB}}$ is the angle between the photon momentum and tangent to the magnetic field line. The explicit formulae for the attenuation length read (Harding et al. 1997)

$$
s=\frac{0.2 \rho_{\mathrm{c}}}{B^{\prime} \epsilon}, \quad \text { if } \quad B \leq 0.1 B_{\mathrm{cr}}
$$

and

$$
s=\frac{2 \rho_{\mathrm{c}}}{B^{\prime}}, \quad \text { if } \quad B \geq 0.1 B_{\mathrm{cr}}
$$


where $\rho_{\mathrm{c}} \approx(4 / 3) \sqrt{c r / \Omega}$ is the radius of curvature.

The case $B \lesssim 0.1 B_{\mathrm{cr}}$

Let us estimate the characteristic thickness of the $\mathrm{SG}, \Delta \xi_{\mathrm{SG}}$, for the case $\chi \approx 0$. Since the acceleration within the SG occurs mostly in the saturation regime (i.e. at high altitudes, where the accelerating $E_{||}$saturates), we can use the following expression for the dimensionless altitude $z_{0}$ (here in units of stellar radius) of pair formation due to CR (cf. eq. [40], for the saturated regime, in HM01) as a function of $\xi$, to estimate the characteristic scale of a SG over $\xi$,

$$
z_{0}=7 \times 10^{-2} \frac{P_{0.1}^{7 / 4}}{B_{12} I_{45}^{3 / 4}} \frac{1}{\xi^{1 / 2}\left(1-\xi^{2}\right)^{3 / 4}},
$$

where $P_{0.1}=P / 0.1 \mathrm{~s}, B_{12}=B_{0} / 10^{12} \mathrm{G}$. In our derivation of formula (18) we employed the same procedure of minimization of the sum of acceleration length and photon mean-free path, as we did in HM98, HM01 and HM02, with the explicit $\xi$ dependence of radius of curvature and accelerating electric field (for $\chi \approx 0$ ) included. The PFF is typically relatively flat over the bulk of the PC (for $0.2 \lesssim \xi \lesssim 0.8$ ), and begins turning up as $\xi$ approaches the rim of the open field line domain and also the magnetic axis. Near the rim the PFF tends to establish at a few stellar radii, so that the colatitude $\xi_{\mathrm{SG}}$ of the beginning of the SG may be estimated via the condition

$$
{\frac{\partial z_{0}}{\partial \xi}}_{l_{\xi=\xi_{S G}}} \sim \lambda
$$

where $\lambda \sim 0.1-0.5$ is a free parameter that can be constrained for the known $\gamma$-ray pulsars by comparison their observed and predicted $\gamma$-ray luminosities. The condition (19) means that the regime of the SG acceleration sets in when the characteristic variation of the PFF altitude with $\xi$-coordinate rescaled by the whole interval of PC $\xi$ values $(\sim 1)$ becomes comparable to $\lambda$ times the stellar radius. Note that formula (19) does not take into account the effect of screening near the $\mathrm{SG}$ and is based therefore on a somewhat underestimated value of $z_{0}$ which results in the underestimation of the SG width. For this reason the most likely value of the parameter $\lambda$ is expected to be in the lower range, say $\lambda \sim 0.1-0.3$. Earlier (see HM98 and HM01) we calculated the shape of the PFF produced at low altitudes by CR photons. In general, those calculations are valid only for the values of $\xi$ where the PFF is relatively flat, because they did not take into account the effect of electric field screening under consideration in the SG region (which will increase the PFF heights there). However, formally, we can extend our calculations for the values of $\xi$ close to 1, so that we can see how the PFF turns up as we approach the rim. Figures $2 \mathrm{a}$ and $2 \mathrm{~b}$ show the height of the PFF produced by CR photons at low altitudes as a function of $\xi$ near the rim region. We present calculations for the values of pulsar spin period $P=0.1 \mathrm{~s}$ (Figure 2a) and $0.2 \mathrm{~s}$ (Figure $2 \mathrm{~b}$ ) and for the discrete values of $\mathrm{B}$, ranging from $0.01 B_{\mathrm{cr}}$ through $0.2 B_{\mathrm{cr}}$. Even though our calculations shown in Figures $2 a$ and $2 b$ are not consistent in the region where the PFF is curving up, they may provide a rough idea about the characteristic width of the SG region. Figures $2 a, b$ show that the typical values of $\xi$ at which the heights of PFF begin to rapidly grow roughly agree with our estimates of the SG width (see eqs. [20] and [22] below). 
Assuming that $\Delta \xi_{\mathrm{sG}} \lesssim 0.3$, from eqs (18) and (19) we find that

$$
\Delta \xi_{\mathrm{sG}} \approx 0.2 P_{0.1}\left(\lambda B_{12}\right)^{-4 / 7} I_{45}^{-3 / 7}
$$

The case $B \gtrsim 0.1 B_{\mathrm{cr}}$

First of all, note that this case was not treated by HM01, but in fact may be important for high-energy pulsars. Using the threshold condition for the pair attenuation length (see eq. [17]), we can write

$$
z_{0}=7 \times 10^{-2} \frac{P_{0.1}^{7 / 4}}{B_{12}^{1 / 2} I_{45}^{3 / 4}} \frac{1}{\xi^{1 / 2}\left(1-\xi^{2}\right)^{3 / 4}} .
$$

so that for the condition (19) we arrive at the estimate,

$$
\Delta \xi_{\mathrm{SG}} \approx 0.2 P_{0.1}\left(\lambda B_{12}^{1 / 2}\right)^{-4 / 7} I_{45}^{-3 / 7}
$$

Note that expressions (20) and (22) match at $B_{12} \sim 1$ and that $\Delta \xi_{\mathrm{sG}}$ tends to increase with decreasing $\lambda$.

By substituting expressions (20) and (22) into formula (12), we can estimate the characteristic electric potential drop as

$$
\Delta \Phi \approx 1.5 \times 10^{12} B_{12}^{\delta}\left(\frac{I_{45}}{\lambda^{8}}\right)^{1 / 7} \mathcal{F} \quad \text { Volts }
$$

where $\delta=-1 / 7$ and $3 / 7$ for the low-B and high-B case, respectively, and

$$
\begin{aligned}
\mathcal{F}= & {\left[\kappa_{0.15}\left(1-\frac{1}{\eta^{3}}\right) \cos \chi+\right.} \\
& \left.3.8 \times 10^{-3}\left(\frac{R_{6}^{7}}{P_{0.1} I_{45}^{2}}\right)^{1 / 2}\left(H(\eta) \sqrt{\eta \frac{f(1)}{f(\eta)}}-H(1)\right) \sin \chi \cos \phi_{\mathrm{pc}}\right]\left(1-\xi_{*}^{2}\right) .
\end{aligned}
$$

The above formula for $\Delta \Phi$ illustrates that the accelerating potential (the Lorentz factor of primary electrons) is only weakly dependent on the pulsar magnetic field, and the dependence on pulsar spin period enters only the second term in expression for $\mathcal{F}$ which is proportional to $\sin \chi$. The strong dependence of the parallel electric field on magnetic field and spin period have been mostly cancelled by the screening parameter $\Delta \xi_{\mathrm{sG}}$ which depends on the pair production mean-free path. The main factors determining $\Delta \Phi$ are thus, the radius and moment of inertia of a NS, and pulsar obliquity angle $\chi$ (note that $\kappa_{0.15}=I_{45} / R_{6}^{3}$ ).

By substituting expressions (20) and (22) into formula (23), we can now estimate the characteristic Lorentz factor of primary electrons, $\gamma=e \Delta \Phi / m_{\mathrm{e}} c^{2}$,

$$
\gamma \approx 4 \times 10^{7}\left(\frac{I_{45}}{\lambda_{0.1}^{8}}\right)^{1 / 7} B_{12}^{\delta}\left(1-\frac{1}{\eta_{\mathrm{acc}}^{3}}\right)
$$


where $\eta_{\mathrm{acc}}$ is the value of $\eta$ corresponding to the characteristic acceleration altitude, and $\lambda_{0.1}=\lambda / 0.1$. For the parameters of a Crab-like pulsar, $B_{12}=5, I_{45}=4$, and assuming $\eta_{\text {acc }}=2$, and $\delta=-1 / 7$ (low-B case), we get

$$
\gamma \sim 3 \times 10^{7} \lambda_{0.1}^{-8 / 7}
$$

In the present analysis the width of the SG in units of dimensionless coordinate $\xi$ is constant as a function of altitude. In reality, it may slightly vary with altitude. For example, it may grow with altitude because the plasma column boundary may be getting less sharp with altitude, so that the efficiency of geometrical screening may slightly degrade (meaning the increase in $\Delta \nu_{\mathrm{SG}}$ ) with altitude. Loosely speaking, the effect of fuzziness of the plasma column boundary at high altitudes can be attributed to the systematic increase in the deviation of the transverse component of the photon mean-free path from the field line toward the magnetic axis.

\subsection{The Gamma-Ray Luminosity per Unit Solid Angle Emitted by the SGs}

Here we calculate the specific high-energy luminosity, $L_{\gamma}\left(\Omega_{\gamma}\right)$, radiated by particles accelerated within the SGs and emitted into the solid angle $\Omega_{\gamma}$.

First, let us calculate the solid angle within which the photons produced by SGs are emitted,

$$
\Omega_{\gamma}=2 \pi \int_{\theta_{\min , \gamma}^{\mathrm{SG}}}^{\theta_{\max , \gamma}^{\mathrm{SG}}} \sin \theta d \theta,
$$

where

$$
\begin{gathered}
\theta_{\mathrm{min}, \gamma}^{\mathrm{SG}} \approx \frac{3}{2} \theta_{0} \sqrt{\eta \frac{f(1)}{f(\eta)}}\left(1-\Delta \xi_{\mathrm{sG}}\right), \\
\theta_{\max , \gamma}^{\mathrm{SG}} \approx \frac{3}{2} \theta_{0} \sqrt{\eta \frac{f(1)}{f(\eta)}} \text { and } \eta \approx \eta_{\gamma} .
\end{gathered}
$$

Thus, from eq. (27) we can get

$$
\Omega_{\gamma} \approx \frac{9}{2} \pi \theta_{0}^{2} \eta \frac{f(1)}{f(\eta)} \Delta \xi_{\mathrm{sG}} \quad \text { ster, } \quad \eta \approx \eta_{\gamma} .
$$

Here $\eta_{\gamma}$ is the radial distance of the high-energy emission site in the SG. Using expressions (10), (13), (30), and the estimated values of $\Delta \xi_{\mathrm{sG}}$ (see eqs [20] and [22]), we get

The case of $B \lesssim 0.1 B_{\mathrm{cr}}$

$$
L_{\gamma}\left(\Omega_{\gamma}\right)=\frac{\varepsilon_{\gamma} L_{\text {prim }}}{\Omega_{\gamma}}=3 \times 10^{34} \varepsilon_{\gamma} L_{\text {sd }, 35}^{3 / 7} P_{0.1}^{5 / 7} R_{6}^{17 / 7} \Lambda\left(\eta_{\gamma}\right) \quad \text { erg } \cdot \mathrm{s}^{-1} \cdot \text { ster }^{-1}
$$

The case of $B \gtrsim 0.1 B_{\mathrm{cr}}$ 


$$
L_{\gamma}\left(\Omega_{\gamma}\right)=\frac{\varepsilon_{\gamma} L_{\text {prim }}}{\Omega_{\gamma}}=3 \times 10^{34} \varepsilon_{\gamma} L_{\mathrm{sd}, 35}^{5 / 7} P_{0.1}^{13 / 7} R_{6}^{5 / 7} \Lambda\left(\eta_{\gamma}\right) \quad \mathrm{erg} \cdot \mathrm{s}^{-1} \cdot \mathrm{ster}^{-1}
$$

where

$$
\begin{aligned}
\Lambda(\eta)= & \frac{f(\eta)}{\eta} \lambda^{-8 / 7} I_{45}^{-6 / 7}\left\{\kappa(1-\kappa)\left(1-\frac{1}{\eta^{3}}\right) \cos ^{2} \chi+\right. \\
& \left.\frac{9}{8} \theta_{0}^{2} H^{2}(1)\left[\frac{H(\eta)}{H(1)} \sqrt{\eta \frac{f(1)}{f(\eta)}}-1\right] \sin ^{2} \chi\right\},
\end{aligned}
$$

and $L_{\mathrm{sd}, 35}=L_{\mathrm{sd}} / 10^{35} \mathrm{erg} \cdot \mathrm{s}^{-1}$. In the above expressions the parameter $\varepsilon_{\gamma}\left(0<\varepsilon_{\gamma}<1\right)$ is the efficiency of transforming the primary energetics into the energetics of high-energy emission. Note that in the above expressions $L_{\gamma}\left(\Omega_{\gamma}\right) \propto L_{\text {sd }}^{3 / 7}$ (low-B case) and $\propto L_{\text {sd }}^{5 / 7}$ (high-B case) simply because $L_{\text {prim }} \propto L_{\text {sd }}^{1 / 7}$ and $\Omega_{\gamma} \propto L_{\mathrm{sd}}^{-2 / 7}$ (low-B case), and $L_{\text {prim }} \propto L_{\mathrm{sd}}^{4 / 7}$ and $\Omega_{\gamma} \propto L_{\mathrm{sd}}^{-1 / 7}$ (high-B case), respectively.

The above expressions for $L_{\gamma}\left(\Omega_{\gamma}\right)$ are equivalent to the observed quantity $\Phi_{\gamma} d^{2}$, where $\Phi_{\gamma}$ is the high-energy bolometric flux observed at the Earth, and $d$ is the distance to the pulsar. Thus, the only free parameters (which may be determined by pair cascade simulations) are the efficiency $\varepsilon_{\gamma}$ and the parameter $\lambda$ determining the SG width. All others are intrinsic properties of the NS. Figure 3 shows the pulsar empirical (solid circles with error bars) and theoretical (upside-down triangles) values of $\Phi_{\gamma} d^{2}$ as a function of spin-down luminosity, $L_{\mathrm{sd}}$. The theoretical values are calculated for the parameters $\varepsilon_{\gamma}=0.3$ and $\lambda=0.1$ (see eq. [31]). Note that parameter $\varepsilon_{\gamma}$ can range from 0.2 to 0.5 in cascade calculation, and $\eta_{\gamma}=3$. In Figure 3 the dashed line represents the absolute upper limit, where the spin-down luminosity is radiated into the unit solid angle, i.e. where $\Phi_{\gamma} d^{2}=L_{\mathrm{sd}} / 1$ ster. One can see that there is a good agreement for most high-energy pulsars except several of the pulsars, Geminga and PSR B0656+14, having low $L_{\text {sd }}$, and for J0218+4232, which is a millisecond pulsar. These pulsars are near or below the CR death line (see HM02, and next Section), and therefore have either very wide SGs or no SGs at all. All other high-energy pulsars depicted in Figure 3 are above their CR death lines and are expected to have SGs.

\subsection{Energetics of Returning Positrons in Slot Gap}

Let us estimate the maximum possible heating luminosity produced by returning positrons within the SG, provided that the PFF establishes at $\eta=\eta_{\mathrm{PFF}}$.

The general equation for the power of returning positrons can be written as (see e.g. MH97, eqs.[76]-[78]; and HM01, eq. [61])

$$
L_{+}=\alpha c \int_{S_{\mathrm{SG}}\left(\eta_{\mathrm{PFF}}\right)} x_{+}\left|\rho_{\mathrm{GJ}}\right| \Phi d S,
$$


where the integration is over the surface cut in a sphere at the radial distance $\eta_{\mathrm{PFF}}$ by the SG boundaries, and $x_{+}=\rho_{+}\left(\eta_{\mathrm{PFF}}\right) /\left|\rho_{\mathrm{GJ}}\left(\eta_{\mathrm{PFF}}\right)\right|$ is the charge density of returning positrons, scaled by the general-relativistic GJ charge density (see eq. [3]).

Let us calculate, for the sake of illustration, the fractional density of returning positrons for $\chi \approx 0$ assuming that $\rho_{+} \approx\left|\rho_{\mathrm{GJ}}\left(\eta_{\mathrm{PFF}}\right)-\rho\right| / 2$. Using formulae (3), (4) and representation of $\kappa$ as $\kappa \approx 0.15 I_{45} / R_{6}^{3}$ (see $\S 2.1$, right after eq. [4]), we get

$$
x_{+} \sim \frac{1}{2} \frac{\kappa}{\left(1-\kappa / \eta_{\mathrm{PFF}}^{3}\right)}\left(1-1 / \eta_{\mathrm{PFF}}^{3}\right) \approx \frac{1}{2} \kappa \approx 0.08 \frac{I_{45}}{R_{6}^{3}} \text { for } \eta_{\mathrm{PFF}} \gtrsim 2,
$$

which means that for a nearly aligned pulsar the maximum fraction of positrons returning from the elevated pair fronts of SGs is independent of the PFF altitude, in contrast to the case of the PFFs establishing at low altitudes (see HM01, HM02) over the bulk core part of the PC.

By inserting $\Phi\left(\eta, \theta, \phi_{\mathrm{pc}}\right)$ and $\rho_{\mathrm{GJ}}(\eta)$ into eq. (34) and performing the integration over $\theta$, in this case (cf. eqs.[28], [29]), from

$$
\theta_{\mathrm{min}}^{\mathrm{SG}}=\theta_{0} \sqrt{\eta_{\mathrm{PFF}} f(1) / f\left(\eta_{\mathrm{PFF}}\right)}\left(1-\Delta \xi_{\mathrm{SG}}\right)
$$

to

$$
\theta_{\max }^{\mathrm{SG}}=\theta_{0} \sqrt{\eta_{\mathrm{PFF}} f(1) / f\left(\eta_{\mathrm{PFF}}\right)}
$$

we get

$$
L_{+}=\frac{1}{4} \kappa^{2}\left(\Delta \xi_{\mathrm{sG}}\right)^{3} L_{\mathrm{sd}}
$$

The case of $B \lesssim 0.1 B_{\mathrm{cr}}$

$$
L_{+} \approx 4 \times 10^{30} \kappa_{0.15}^{2} \lambda^{-12 / 7} P_{0.1}^{-3 / 7} I_{45}^{5 / 7} R_{6}^{-6 / 7} L_{\mathrm{sd}, 35}^{1 / 7} \quad \mathrm{erg} \cdot \mathrm{s}^{-1} .
$$

The case $B \gtrsim 0.1 B_{\mathrm{cr}}$

$$
L_{+} \approx 4 \times 10^{30} \kappa_{0.15}^{2} \lambda^{-12 / 7} P_{0.1}^{9 / 7} I_{45}^{5 / 7} R_{6}^{-24 / 7} L_{\mathrm{sd}, 35}^{4 / 7} \quad \mathrm{erg} \cdot \mathrm{s}^{-1}
$$

The contribution from heating by positrons returning from high altitudes of SGs should be taken into account in the estimates of the PC surface temperatures (see HM01, HM02)

It is interesting to note, that the condition of turning-on of the SG acceleration in pulsars should be tightly related to the pair death line condition determined by the pair-formation above the central part of the PC (see e.g. HM02 for detailed discussion), simply because the very existence of the gap is determined by establishing the pair plasma column and the screening of $E_{\|}$ by pairs. The analytic pair CR death line (see eq. [12] in HMZ02) reads

$$
\log \dot{P} \geq\left\{\begin{array}{ll}
\frac{21}{8} \log P-\frac{7}{4} \log \bar{f}_{\text {prim }}^{\min }-\Delta_{\mathrm{I}}^{(\mathrm{CR})}(R, I)-14.6 & P \lesssim P_{*}^{(\mathrm{CR})} \\
\frac{5}{2} \log P-2 \log \bar{f}_{\text {prim }}^{\min }-\Delta_{\mathrm{II}}^{(\mathrm{CR})}(R, I)-15.4 & P \gtrsim P_{*}^{(\mathrm{CR})},
\end{array},\right.
$$


where

$$
\Delta_{\mathrm{I}}^{(\mathrm{CR})}(R, I)=1.5\left(\log I_{45}-6.3 \log R_{6}\right)
$$

and

$$
\Delta_{\mathrm{II}}^{(\mathrm{CR})}(R, I)=1.5\left(\log I_{45}-7.3 \log R_{6}\right)
$$

are corrections for the NS equation of state, and

$$
P_{*}^{(C R)}=0.1 \cdot B_{12}^{4 / 9}
$$

is the value of pulsar spin period separating the regime of acceleration of primaries where the $E_{\|}$ is increasing with altitude (unsaturated) from the regime where $E_{\|}$remains practically constant (saturates). In eq. (41) $\bar{f}_{\text {prim }}^{\min }$ is the minimum efficiency of converting spin-down luminosity into the luminosity of the primary beam needed for pair formation (see HM02 and HMZ02 for details). According to our calculation performed in HM02 the value of parameter $\bar{f}_{\text {prim }}^{\min }$ ranges from 0.2 to 0.5 . For the parameters of a canonical NS and for $\bar{f}_{\text {prim }}^{\min }=0.2$, we get

$$
\log \dot{P} \gtrsim 2.5 \log P-14
$$

as the required condition for $\mathrm{SGs}$ to form.

Note also that the relationship between the pulsar death line and the regime of SG acceleration may be justified only for the CR-produced pair front. Even though the SGs may still be formed in the case of the ICS pair production above the bulk of the PC, the density of pair plasma may not be sufficient to guarantee the efficiency of the geometrical screening. In this case the effective parameter $\Delta \nu_{\mathrm{SG}}$ may significantly differ from that calculated in this paper, including the case where it approaches 1 (no geometrical screening within the $S G$ ). Our previous calculation of the ICS PFFs indicates (see e.g. HM01, HM02) that this situation may well occur in some pulsars that are slightly below the death line (e.g. in millisecond and relatively old pulsars). In this paper we do not intend to discuss these non-ideal SGs.

\section{Pair Cascade Simulation}

Before we discuss our numerical simulation it is worth mentioning that there is a long history associated with the "prototype" of a SG model, the pure geometrical model of pulsar emission known as the hollow cone model. By analyzing the smooth variation of the direction of linear polarization within the radio pulsar pulse window Radhakrishnan \& Cooke (1969) and Radhakrishnan (1969) realized that these observations suggested that pulsars must be magnetized NSs which produced relativistic particles from near the magnetic PCs. Komesaroff (1970) showed that if the radio emission is due to $\mathrm{CR}$ then the pulsar beam must be a hollow cone. The double pulses observed in some pulsars, the pulse width, polarization, as well as the spectrum of pulses found a natural explanation within this model. 
In order to explore the characteristics of high-energy emission produced by particles accelerated in the SG, we have carried out Monte Carlo simulations of the pair cascades initiated by primary electrons accelerated by the $E_{\|}$in the gap. This calculation is very similar to the pair cascade simulation described by DH96. In that paper, primary electrons were accelerated above a pulsar PC by a simple model $E_{\|}$described by starting and terminating altitudes above the NS surface, a constant term and a linear term. These parameters were adjusted to produce both a sufficient final Lorentz factor of the primary electrons and a wide enough gamma-ray beam geometry to explain the EGRET spectrum and pulse profile of the Vela pulsar. DH96 found that a $E_{\|}$starting altitude of 2 stellar radii was required, but no physical justification was provided to support this value. DH96 also artificially enhanced the primary particle flux at the $\mathrm{PC}$ rim in order to produce the hollow emission cone, narrow pulses and low level of bridge emission necessary to model the Vela profile.

In the present study, we use the SG $E_{\|}=-\nabla \Phi$ derived from the potential in eq. (12). The acceleration rate as a function of distance is

$$
\frac{d \gamma}{d s}=\frac{e E_{\|}}{m_{\mathrm{e}} c^{2}} \approx 54 \frac{R_{6}^{2} B_{12}^{\delta}}{\left(\lambda^{4} I_{45}^{3}\right)^{2 / 7}}\left(\frac{\kappa}{\eta^{4}} \cos \chi+\frac{1}{4} \theta_{0} \eta^{-1 / 2} \sin \chi \cos \phi_{\mathrm{pc}}\right)\left(1-\xi_{*}^{2}\right),
$$

where $\delta$ is defined right after eq. (23). In this formula, for the sake of illustration, we pull out the general-relativistic correction from the second term to get its simple explicit $\eta$-dependence. Even though in most cases the second term in the above equation is less than the first one, in our numerical calculations we use the exact formula for $E_{\|}$based on expression (12). The first term in parentheses is the contribution to acceleration from the inertial frame-dragging effect, where $\kappa \approx 0.15 I_{45} / R_{6}^{3}$ is the general relativistic parameter that depends on the NS compactness. The second term is the contribution from the field-line curvature, similar to the accelerating field of AS79. Factors of order one have been omitted from this term in order to simplify the expression in the potential of eq. (12). Note that the SG acceleration rate given above is only mildly dependent on $\mathrm{B}$ and $\mathrm{P}$. We assume that primary electrons begin their acceleration at the NS surface on a central field line of the $\mathrm{SG}, \xi_{*}=0$. We follow the electrons as they gain energy and follow their CR photons through the local magnetic field, accumulating the probability for creating an electron-positron pair. If the $\mathrm{CR}$ photons pair produce, the synchrotron emission of the created pair is simulated and the synchrotron radiation photons are followed in the same way to determine if they pair produce or escape. Details of the cascade simulation may be found in DH96 and Harding et al. (1997). We ignore inverse Compton emission of the primaries and pairs and photon splitting of the CR photons.

To illustrate the features of the SG acceleration and cascades, we use the period $\left(P_{0.1}=0.33\right)$ and surface magnetic field $\left(B_{12}=5\right)$ of the Crab pulsar for the case $B<0.1 B_{\mathrm{cr}}$ in eq. (46). A stellar radius of $16 \mathrm{~km}$ is assumed. Although the $S G$ is a region devoid of pair plasma near the surface, full pair cascades can develop above the PFFs that form at high altitudes along field lines near the PC rim. In contrast to the pair cascades that form in the core of the PC and terminate within a stellar radius of the NS surface, the pair cascades from particles accelerated in the SG 
develop over a larger distance and the screening of the electric field by the pairs thus requires a much larger distance. The primary electrons reach Lorentz factors of $10^{7}$ in the SG before becoming radiation-reaction limited. However, the pair cascades begin at lower Lorentz factors but the pair multiplicity builds up more slowly in comparison to pair cascades in the PC core (see HM01), so that the screening distance in the SG is relatively large and can be one or two stellar radii. We find that the pair cascade starts at an altitude of $0.5-1.0$ stellar radii and continues up to 3-4 radii. Since the bulk of the escaping high-energy photons are produced between 2 and 4 NS radii, the opening angle of the high-energy beam is much larger (about 20 degrees for the Crab period) than for near-surface $\mathrm{PC}$ emission.

Figure 4 shows plots of the distribution on the sky of escaping cascade radiation above 100 $\mathrm{keV}$ as a function of phase, $\phi$, and viewing angle with respect to the rotation axis, $\zeta$, for different assumed values of the inclination. For relatively small inclination angles that are comparable to the high- energy beam opening angle, emission can be seen over a large range of observer angles. As the inclination angle decreases, the hollow cone pattern becomes smaller and emission is visible over a smaller range of viewing angles. Also at smaller inclination angles, the emission hollow cone is complete because particle acceleration takes place at all phase angles around the magnetic pole. This is a result of the fact that the frame dragging term in the potential is independent of $\phi_{\mathrm{pc}}$. At larger inclination angles, our results show that the emission occurs over only part of the hollow cone. This is due to the $\sin \chi \cos \phi_{\mathbf{p c}}$ dependence of the second term in the potential due to the "favorably curved" field line acceleration that was the only source of accelerated particle in Arons' potential. At large inclination angles this part of the potential dominates as the frame dragging term is declining. Because the favorably curved field lines from the two poles accelerate particles in opposite hemispheres, it is impossible for a single observer to view both poles of the pulsar in this model unless the high-energy beam opening angle is unrealistically large. Instead, at large viewing angles an observer would see a single narrow pulse.

Pulse profiles are formed by slicing across the phase plots in Figure 4 at a constant viewing angle. Examples of pulse profiles at several different inclination and viewing angles are shown in Figure 5. It is evident that widely spaced double-peaked profiles can result at relatively small inclination by slicing through the middle of the hollow cone. Profiles with two pulses separated by more than 100 degrees, as are seen from the Crab, Vela and Geminga, can be formed when the inclination angle is comparable to the beam opening angle (about 20 degrees in this model for the Crab). Broad single pulses, as seen from PSR B1509-58, may be produced when the observer cuts along the edge of the cone. As the inclination angle increases, the maximum pulse separation decreases and the pulses themselves also become narrower. Bridge emission as seen in Vela and Geminga high-energy profiles (and in the Crab profile around $1 \mathrm{MeV}$ ) is not produced in this calculation since we have modeled only cascades and emission from the SG, which produces a pure hollow cone beam. The core cascades will produce additional emission on field lines interior to the $\mathrm{SG}$ which will fill in the profile between the two pulses and produce bridge emission. Emission from the full $\mathrm{PC}$ will be included in future modeling. Emission can be seen in this model at all 
phases because primary electrons will continue to radiate curvature emission at higher altitudes (all the way to the light cylinder) above the termination of the pair cascade. This is the "off-beam" emission (Harding \& Zhang 2001) that will be much softer than the on-beam emission from the cone.

Our cascade simulation includes aberration of the emitted photons as well as delay times between photons emitted at different altitudes. Both of these effects become more important at the higher altitudes of the SG cascades and will produce asymmetries in the pulse profiles, especially for fast rotators. For example, aberration will produce a pair attenuation cutoff at lower energies for the pulse at the leading edge of the profile because the photon emitted at the leading edge attain large enough angles to the magnetic field to produce a pair in a shorter distance than photons emitted at the trailing edge. This effect was studied recently by Dyks \& Rudak (2002), who found that the observed disappearance of the first pulse in double-peaked profiles of the Crab, Vela and Geminga pulsars could be produced if the photons were emitted at altitudes of several stellar radii above the surface. Emission from the SG cascades will therefore naturally produce this effect.

\section{Discussion and Conclusions}

In this paper we revise the regime of electron acceleration within the SG regions above pulsar PCs. We have demonstrated that our treatment of acceleration within the SG allows us to address the longstanding issues of $\gamma$-ray production at high altitudes in the pulsar inner magnetosphere, interpretation of multifrequency light curves of pulsars, and emission energetics. The fundamental differences between the SG model presented in this paper and the original SG model of AS79 are

1. Our model takes into account the effect of electric field screening within the gap caused by the narrowness of the space between the gap equipotential surfaces (effect of nearly parallel conducting boundaries on the accelerating electric field).

2. Our model incorporates the effect of general-relativistic frame dragging, which in most cases determines the PC acceleration properties. As a consequence of this, our SG model is operable for all azimuthal angles around the PC and for all pulsar obliquities, whereas the classical Arons-Scharlemann model operates only for the so-called "favorably curved" (curved toward the rotation axis) field lines, and for nearly orthogonal rotators.

3. We consider the high-energy emission from pair cascades at the inner edge of the SG while Arons $(1983,1996)$ considered only the CR from primaries in the SG.

The first two effects make it possible to initially boost the electron acceleration and then prolong it up to very high altitudes (from few to several stellar radii) where they generate pair-producing CR photons. As a result of pair formation at high altitudes, the high-energy 
photons get emitted into a beam that is larger than the solid angle pertaining to the SG itself. These effects also enable the functioning of SGs in all pulsars which are above the CR pair-formation death lines (see HMZ02 for the details of our most recent death-line calculations). The second property significantly enlarges the allowed parameter space for the pulsar obliquity and viewing angles, in particular to small inclination and nearly aligned rotators where the original SG model failed to produce any acceleration. Within the SG model proposed in this paper both double and single high-energy pulses are possible, and in the case of double pulses the separation between the peaks may be anywhere from near zero to 180 degrees. The asymmetry of double-peaked high-energy pulses (one peak is higher than other), and misalignment of $\gamma$-ray light curves with the X-ray light curves may be attributed to the effect of photon aberration which becomes important for high-energy photons generated at high altitudes. Some other effects, such as e.g. effect of NS equation-of-state and effects of very strong stellar magnetic field will be discussed separately in our subsequent publications. Also, we plan to investigate the details of formation of high-energy spectra in SGs of pulsars and perform comparison with the observational data.

In this paper we calculated the predicted high-energy luminosity per unit solid angle from the SG of pulsars as a function of pulsar spin-down luminosity. Our theoretical calculations agree well with the observational values for $\gamma$-ray pulsars. Given the fact that our model has only two major uncertainties, such as the uncertainty in efficiency of conversion of primary energetics into the energetics of $\gamma$-ray emission and uncertainty of our calculation of SG width, the agreement is rather remarkable. Note that the uncertainty of our SG width calculation translates into the uncertainty of order of a few for our calculation of high-energy luminosity per unit solid angle which is comparable to a typical uncertainty of the observations. Our calculations depicted in Figure 3 were performed for the efficiency of conversion of primary energetics into the energetics of high-energy emission $\varepsilon_{\gamma}=0.3$, which does not seem to be unreasonable.

The SG cascades will produce a component of electron-positron pairs at high altitudes, in addition to and distinct from the pair component produced by the low-altitude core cascades. One might speculate that these two pair components could be the origin of the core and conal radio emission components that have been identified from radio morphology studies (e.g. Rankin 1993, Lyne \& Manchester 1988). It will be interesting to explore the properties of these two pair components, which may have different characteristics.

We presented our illustrative calculations of $\gamma$-ray light curves and double-peaked pulse profiles for the parameters of the Crab pulsar. We believe that our simulated high-energy light curves and pulse profiles can be used to constrain the obliquity and viewing angles for $\gamma$-ray pulsars. We plan to perform detailed and extensive simulations of this kind in the future. We also plan to perform comparative analysis of two-pole and one-pole models for selected pulsars with available multifrequency light curves and indirect studies of their viewing and obliquity angles. 
We would like to thank Matthew Baring and Bing Zhang for discussions and many helpful

comments on the manuscript. We also acknowledge support from the NASA Astrophysics Theory Program.

\section{REFERENCES}

Arons, J. 1981, in IAU Symp. No. 95, 'Pulsars', eds W. Sieber and R. Wielebinski (Dordrecht: Reidel), p. 69.

Arons, J. 1983, ApJ, 266, 215 (A83).

Arons, J. 1996, Astron. Astroph. Suppl. Ser., 120, 49 (A96).

Arons, J., \& Scharlemann, E. T. 1979, ApJ, 231, 854 (AS79).

Becker, W. \& Aschenbach, B. 2002, in 'Neutron Stars, Pulsars and Supernova Remnants', ed. W. Becker, H. Lesche \& J. Trumper (MPE Report 278), p. 63 (astro-ph/0208466).

Cheng, K. S., Ho, C., \& Ruderman, M. A. 1986, ApJ, 300, 500.

Daugherty, J. K., \& Harding, A. K. 1996, ApJ, 458, 278 (DH96).

Deutsch, A. 1955, Ann. d'Ap., 18, 1.

Dyks, J. \& Rudak, B. 2002, A \& A, 393, 511.

Harding, A. K., Baring, M. G., \& Gonthier, P. G. 1997, ApJ, 476, 246.

Harding, A. K., \& Muslimov, A. G. 1998, ApJ, 508, 328 (HM98).

Harding, A. K., \& Muslimov, A. G. 2001, ApJ, 556, 987 (HM01).

Harding, A. K., \& Muslimov, A. G. 2002, ApJ, 568, 862 (HM02).

Harding, A. K., Muslimov, A. G., \& Zhang, B. 2002, ApJ, 576, 366 (HMZ02).

Harding, A. K. \& Zhang, B. 2001, ApJ, 548, L37.

Hirotani, K. \& Shibata, S. 2001, MNRAS, 325, 1228.

Komesaroff, M. M. 1970, Nature, 225, 612.

Landau, L. D., \& Lifshitz, E.M. 1984, The Electrodynamics of Continuous Media (Pergamon, Oxford).

Lyne, A. G. \& Manchester, R. N. 1988, MNRAS, 234, 477.

Muslimov, A. G., \& Tsygan, A. I. 1992, MNRAS, 255, 61 (MT92).

Muslimov, A. G., \& Harding, A. K. 1997, ApJ, 485, 735 (MH97).

Radhakrishnan, V. 1969, Proc, Astron. Soc. Austr., 1, 254.

Radhakrishnan, V., \& Cooke, D. J. 1969, Ap. Lett., 3, 225.

Rankin, J. M. 1993, ApJ, 405, 285. 
Rudak, B., Dyks, J. \& Bulik, T. 2002, published in Proc. of the 270. WE-Heraeus Seminar "Neutron Stars, Pulsars and Supernova Remnants", Bad Honnef (Germany), Jan. 21-25, 2002, eds. W. Becker, H. Lesch and J. Truemper, in press (astro-ph/0206101).

Thompson, D. J., 2001, in High-Energy Gamma-Ray Astronomy, ed. F. A. Aharonian \& H. J. Volk, (AIP, New York), p. 103. 


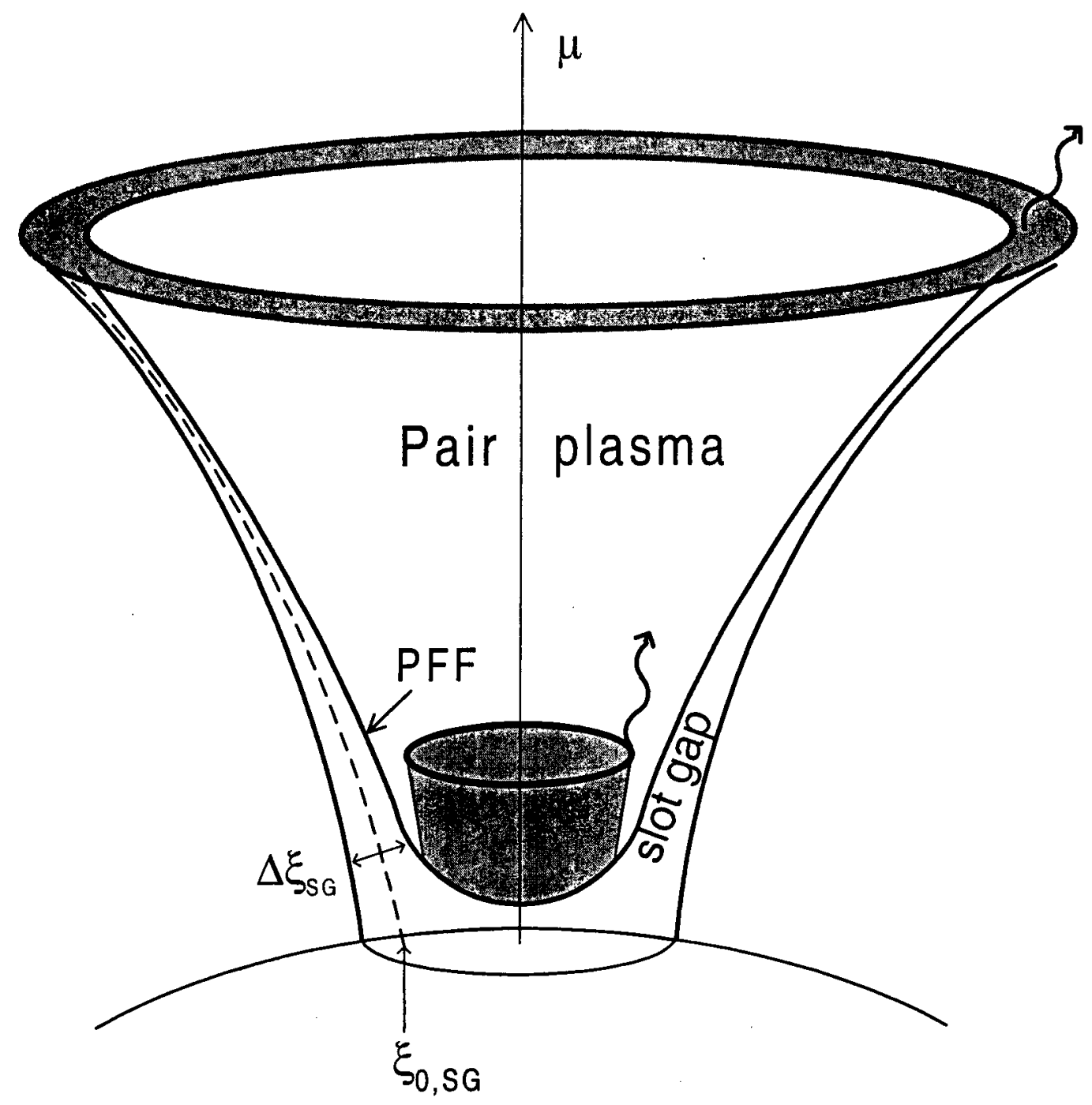

Fig. 1.- Schematic illustration of polar cap geometry, showing the outer boundary of the open field line region (where $E_{\|}=0$ ) and the curved shape of the pair formation front (PFF) which asymptotically approaches the boundary at high altitude. The slot gap exists between the pair plasma which results from the pair cascades above the PFF and the outer boundary. A narrow beam of high-energy emission originates from the low-altitude cascade on field line interior to the slot gap. A broader, hollow-cone beam originates from the high-altitude cascade above the interior edge of the slot gap. $\Delta \xi$ is the slot gap thickness (see text) and $\theta_{0, S G}$ is the colatitude at the center of the slot gap. 

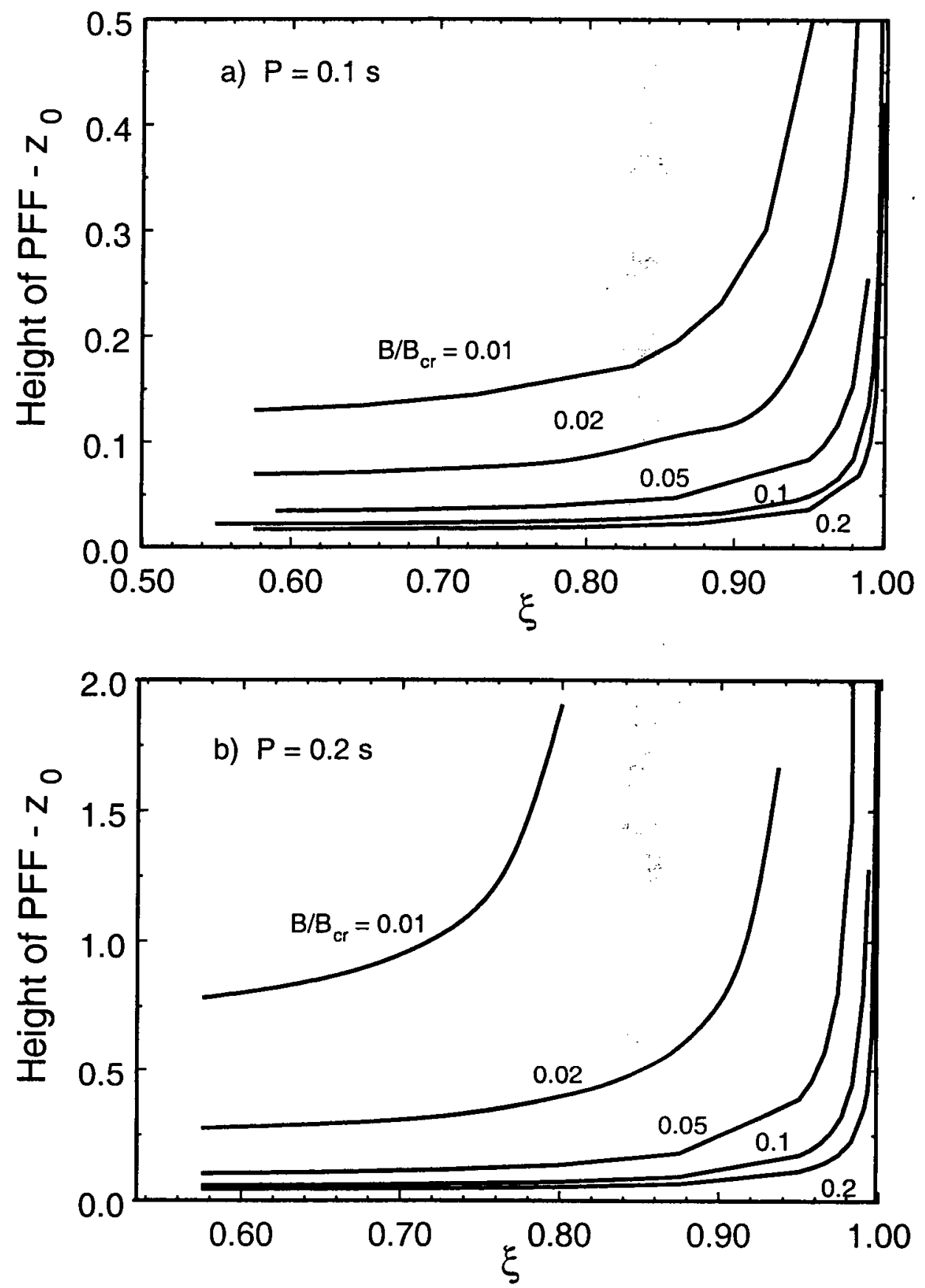

Fig. 2.- Height of the pair formation front for curvature radiation photons above the neutron star (NS) surface $z_{0}$, in units of NS radius $\mathrm{R}$, as a funtion of magnetic colatitude in units of polar cap half-angle, $\xi \equiv \theta / \theta_{0}$, for different values of surface magnetic field strength $\mathrm{B}$, in units of critical field $B_{\mathrm{cr}}$ and $\chi=30^{\circ}$. 


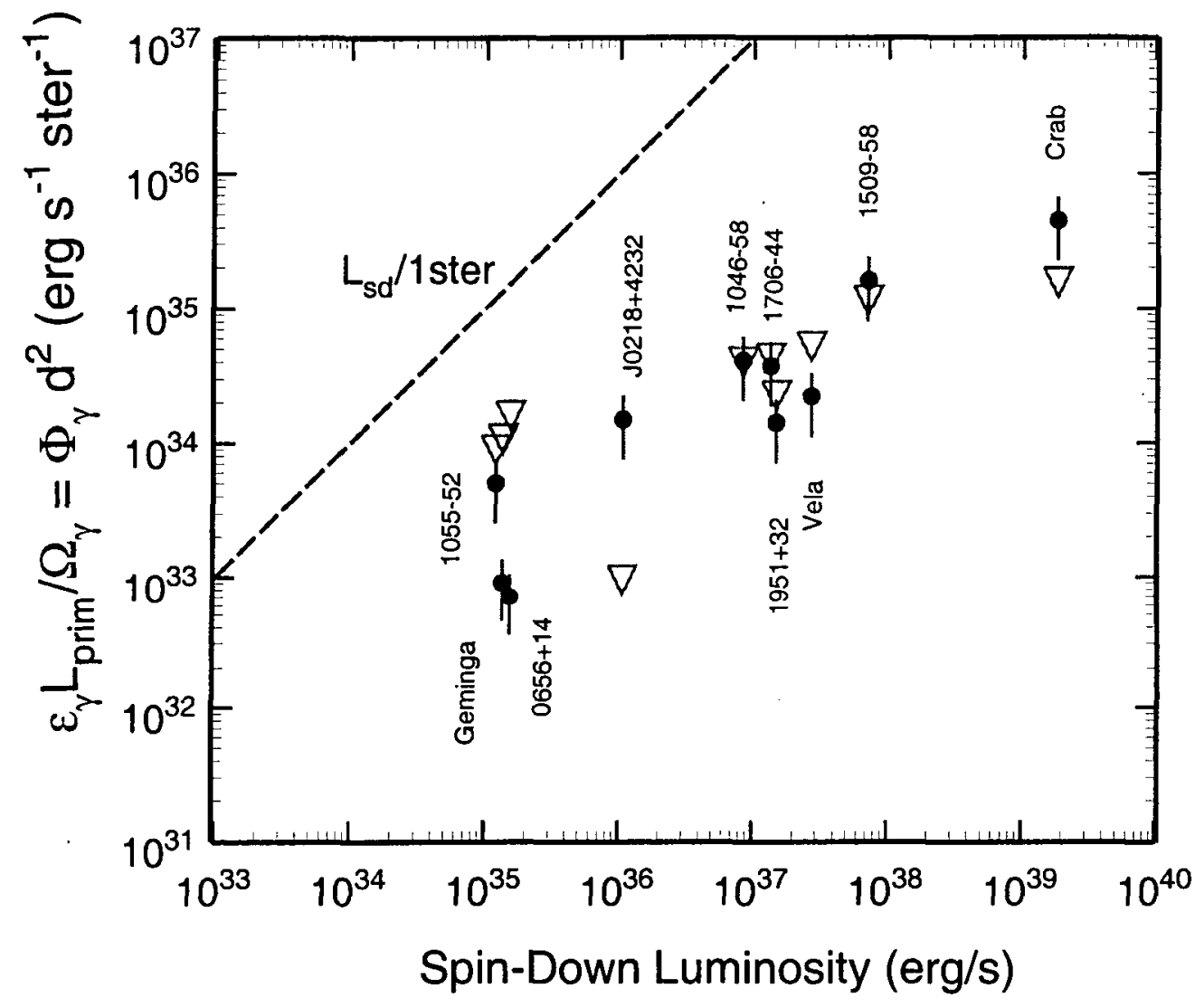

Fig. 3.- Observed flux above $1 \mathrm{keV}, \Phi_{\gamma}$, times distance squared (from Thompson (2001)) (solid circles) and theoretical values of specific high-energy luminosity from the slot gap, $\varepsilon_{\gamma} L_{\text {prim }} / \Omega_{\gamma}$ from eq. (31) (upside-down triangles) vs. spin-down luminosity for known $\gamma$-ray pulsars. An efficiency of $\varepsilon_{\gamma}=0.3$ was assumed. Also $\lambda=0.1, \eta_{\gamma}=3$ and the stellar parameters $R_{6}=1.6$ and $I_{45}=4$ were used. 

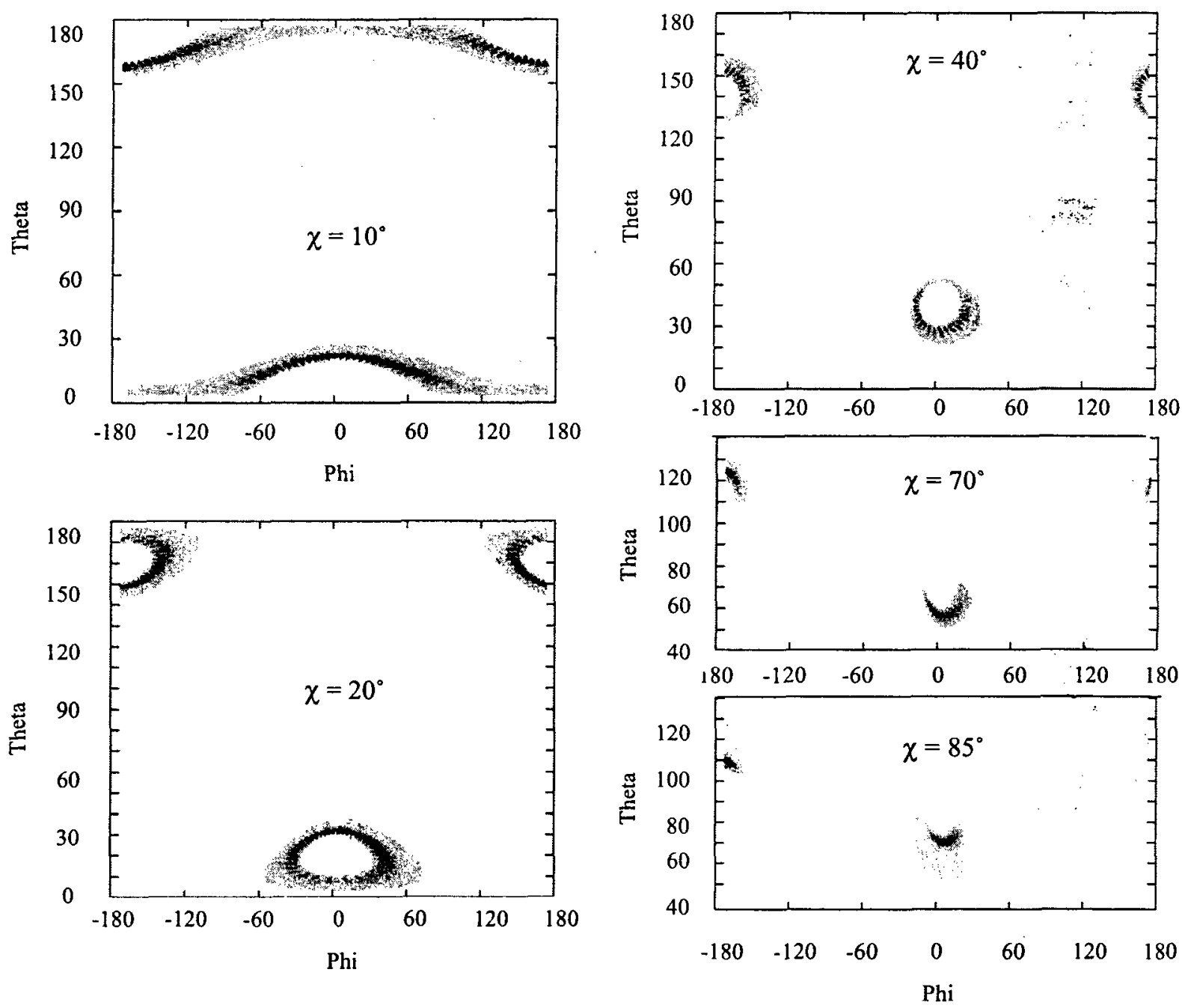

Fig. 4.- Angular distribution of photon emission above $100 \mathrm{keV}$ from pair cascades above the slot gap (using a linear 10-level gray scale), for different values of inclination angle $\chi$. Theta and phi are polar and azimuthal angles with respect to the pulsar rotation axis. Cascade simulations assumed values, $P=33 \mathrm{~ms}, B=5 \times 10^{12} \mathrm{G}$, of the Crab pulsar. Extended faint tracks are high-altitude curvature radiation from primary electrons above the cascade region and are an artifact of the resolution in magnetic phase angle $\phi_{\mathrm{pc}}$ around the polar cap. 

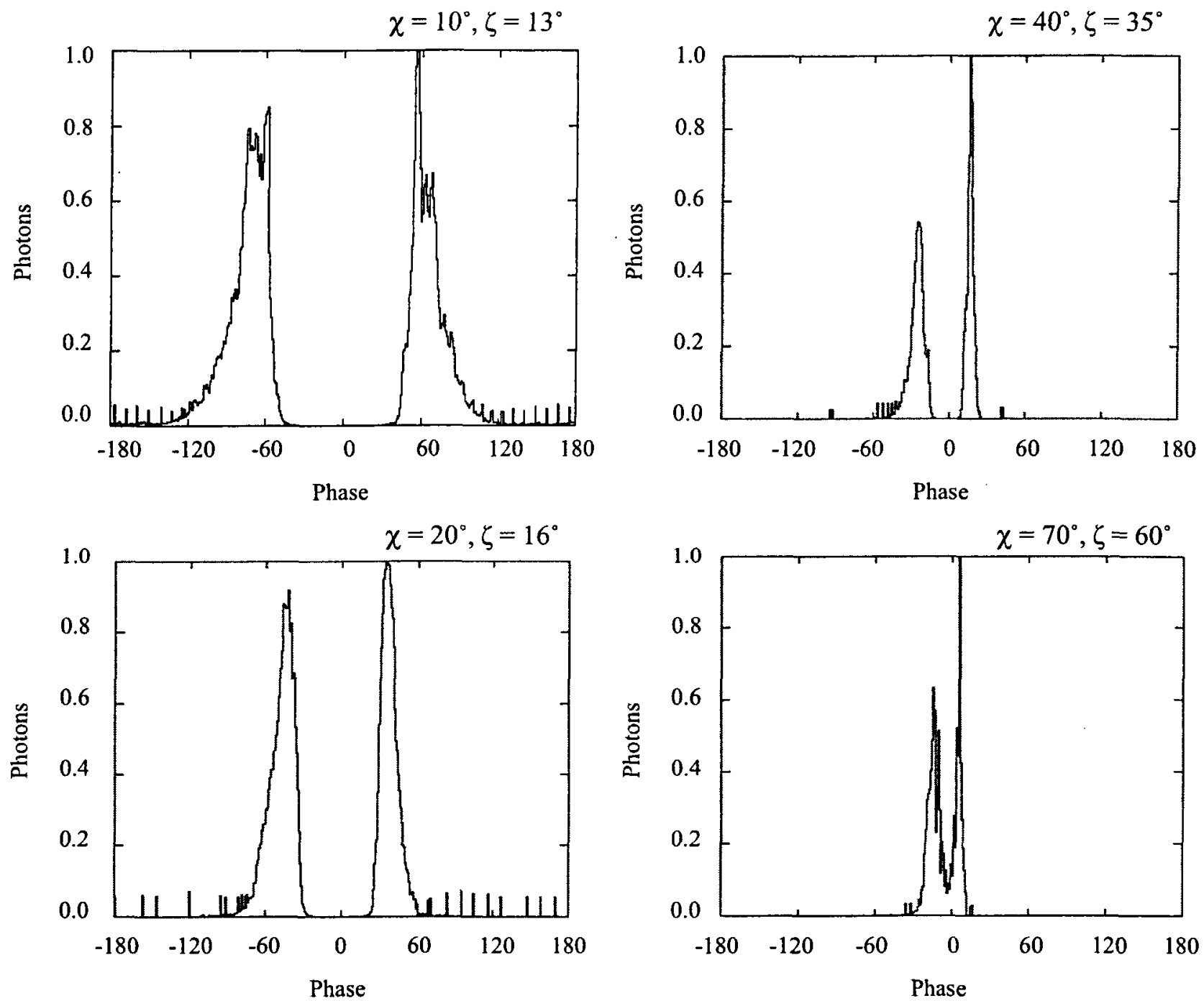

Fig. 5.- Theoretical pulse profiles of photon emission above $100 \mathrm{keV}$ from the slot gap pair cascade at various viewing angles, $\zeta$, generated by making cuts through the angular distributions shown in Figure 4 at constant phase angle theta. The spikes on the leading and trailing edges of the peaks in some profiles are artifacts of the resolution in magnetic phase angle $\phi_{\mathrm{pc}}$ around the polar cap. 